\title{
Diagnostics of Possibility to Increase Prediction of Dynamic of Charge Carriers in A P-N-Junction with Optimized Distributions of Dopants
}

\author{
E.L. Pankratov ${ }^{1, *}$, E.A. Bulaeva ${ }^{2}$ \\ ${ }^{1}$ Nizhny Novgorod State University, 23 Gagarin avenue, Nizhny Novgorod, 603950, Russia \\ ${ }^{2}$ Nizhny Novgorod State University of Architecture and Civil Engineering, 65 Il'insky street, Nizhny Novgorod, 603950, Russia \\ *Corresponding Author: elp2004@mail.ru
}

Copyright $@ 2013$ Horizon Research Publishing All rights reserved.

\begin{abstract}
It has been recently shown, that manufacturing a diffusion-junction rectifier in a multilayer structure at optimal relation between annealing time, materials and thicknesses of layers of the structure gives us possibility to increase sharpness of $p$ - $n$-junctions and to increase homogeneity of dopant distribution in enriched area. In this paper we estimate distributions of concentrations of charge carriers in the $p-n$-junction. At the same time we introduce an analytical approach to estimate the distributions. The approach gives us possibility to take into account spatiotemporal variations of properties of materials and several effects at one tine (diffusion of charge carriers et all), which recently have taken into account independently from each other.
\end{abstract}

Keywords Optimized Diffusion-Junction Rectifier, Dynamics of Charge Carriers; Analytical Approach of Modeling

\section{Introduction}

Manufacturing of new types of microelectronic devices and optimization of old devices are the basis of manufacturing of new integrated circuits [1-5]. For the both cases it is necessary to analyze physical processes during manufacturing of microelectronic devices and during operating of the devices [1-5]. In this paper we consider a multiplayer structure (MS), which consist of a substrate (S) and an epitaxial layer (EL). The structure is presented in Fig. 1. Let us consider a dopant, which has been infused in the EL. After annealing with the optimal continuance one can obtain increasing of sharpness of $p$ - $n$-junction [6-10]. Main aim of the present paper is estimation of distributions of concentrations of charge carriers in the $p$ - $n$-junction after the optimization. Accompanying aim of the present paper is choosing of mathematical apparatus to estimate the distributions. Qualitative structure of $\mathrm{p}$-n-junction is presented in the Fig. 2. In this figure and in future in the text we introduce the following markings: $\varphi \mathrm{k}$ is the contact difference of potentials; $U(t)$ is the applied difference of potentials; $L n$ and -Lp are boundaries of area $p-n$-junction.

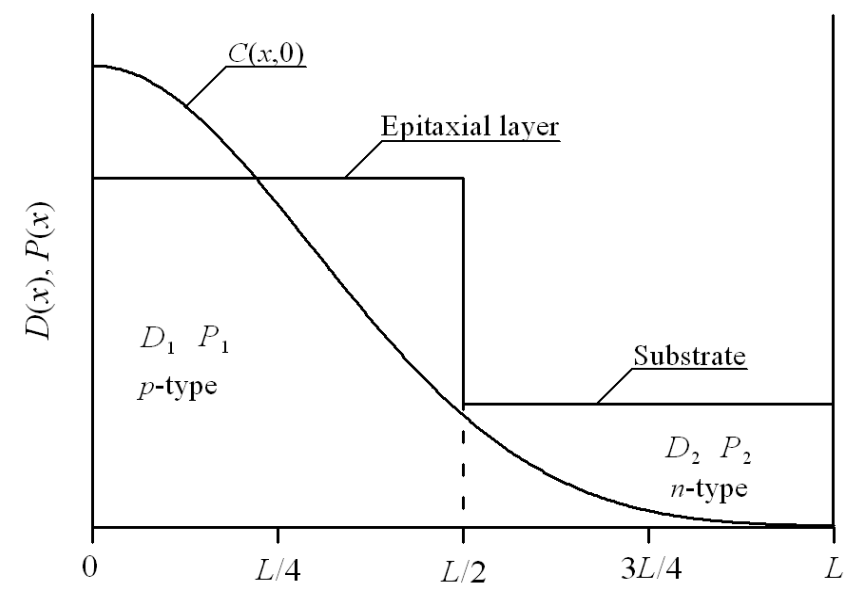

Figure 1. Initial distribution of dopant and types of conductivity of multiplayer structure. $D_{i}$ и $P_{i}$ are the dopant diffusion coefficients and limits of solubility in layers of the multiplayer structure.

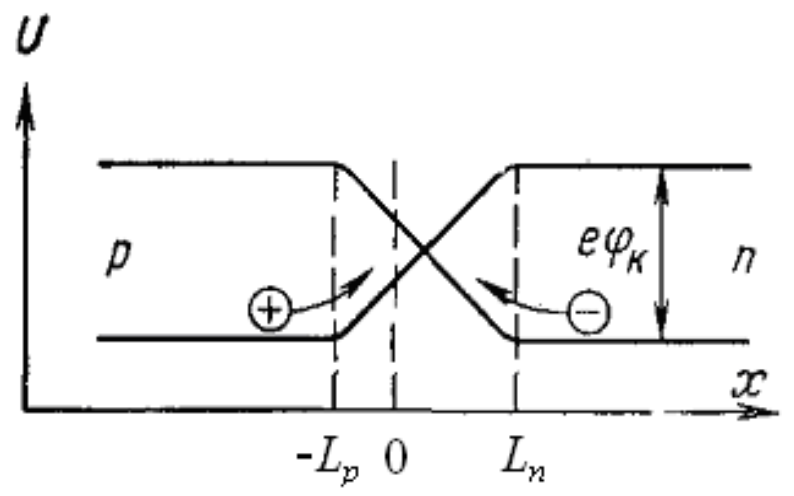

Figure 2. Qualitative structure of $p-n$-junction.

Let us describe spatiotemporal distributions of concentrations of charge carriers in the $p$ - $n$-junction by the following system of equations 


$$
\left\{\begin{array}{r}
\frac{\partial n(x, t)}{\partial t}=G+\frac{\partial}{\partial x}\left[D_{n} \frac{\partial n(x, t)}{\partial x}\right]-\frac{\partial}{\partial x}\left\{\mu_{n} n(x, t) \frac{\partial\left[\varphi(x, t)+\varphi_{h}(x, t)\right]}{\partial x}\right\}- \\
\frac{\partial p(x, t)}{\partial t}=G+\frac{\partial}{\partial x}\left[D_{p} \frac{\partial p(x, t)}{\partial x}\right]+\frac{\partial}{\partial x}\left\{\mu_{p} p(x, t) \frac{\partial\left[\varphi(x, t)+\varphi_{h}(x, t)\right]}{\partial x}\right\}- \\
-k_{n p}\left[n(x, t) p(x, t)-n_{0} p_{0}\right]
\end{array}\right.
$$

Here $\rho(x, t) \mathrm{cm}^{-3}(\rho=n, p)$ are the spatiotemporal ( $x \mathrm{~cm}$ is the coordinate, $t s$ is the time) distributions of concentrations of electrons (for $\rho=n$ ) and holes (for $\rho=p$ ); $\rho_{0}$ are the equilibrium distributions of charge carriers; $D_{\rho} \mathrm{cm}^{2} \mathrm{~s}^{-1}$ are the diffusion coefficients of charge carriers; $\mu_{\rho} \mathrm{cm}^{2} V^{1} \cdot s$ are the mobilities of charge carriers; $\varphi(x, t)$ is the distribution of potential in area of space charge; $\varphi_{h}(x, t) V$ is the potential barrier of heterojunction; $\mathrm{G} \mathrm{cm}^{-3} \mathrm{~s}^{-1}$ is the velocity of generation of electron-hole pairs; $k_{n p} \mathrm{~cm}^{3} s^{-1}$ is the parameter of recombination. Boundary and initial conditions for the system of equation (1) could be written as

$$
\begin{array}{r}
n\left(L_{n}, t\right)=n_{n}(t), n\left(-L_{p}, t\right)=n_{p}(t), p\left(L_{n}, t\right)=p_{n}(t), p\left(-L_{p}, t\right)=p_{p}(t), \\
n(x, 0)=n_{0}(x), p(x, 0)=p_{0}(x) .
\end{array}
$$

Let us determine distribution of potential in area of space charge as solution of the following Poisson equation [4]

$$
\frac{\partial^{2} \varphi(x, t)}{\partial x^{2}}=e \frac{N_{a}(x, t)-N_{d}(x, t)}{\varepsilon \varepsilon_{0}},
$$

where $N_{a}(x, t) \mathrm{cm}^{-3}$ and $N_{d}(x, t) \mathrm{cm}^{-3}$ are the spatiotemporal distributions of acceptor and donor dopants, respectively; $e$ is the elementary charge; $\varepsilon$ is the electric inductivity of materials; $\varepsilon_{0} \approx 8,85 \cdot 10^{-12} \mathrm{~F} / \mathrm{m}$ is the dielectric constant. Boundary conditions for the Poisson equation could be written as

$$
\varphi\left(L_{n}, t\right)=\varphi_{k}+U(t), \varphi\left(-L_{p}, t\right)=U(t),
$$

where $U(t) V$ is the applied difference of potentials.

Farther let us consider single-carrier ionization, when $N_{a}(x, t)=p(x, t)$ and $N_{d}(x, t)=n(x, t)$. In this situation

$$
\frac{\partial^{2} \varphi(x, t)}{\partial x^{2}}=e \frac{p(x, t)-n(x, t)}{\varepsilon \varepsilon_{0}} .
$$

One can obtain solution of the above equation with boundary conditions (4) in the following form

$$
\begin{gathered}
\varphi(x, t)=\varphi_{k}+U(t)+\frac{x-L_{n}}{L_{p}+L_{n}}\left\{\varphi_{k}+U(t)-\frac{e}{\varepsilon \varepsilon_{0}} \int_{-L_{p}}^{L_{n}}\left(L_{n}-v\right)[p(v, t)-n(v, t)] d v\right\}- \\
-\frac{e}{\varepsilon \varepsilon_{0}} \int_{x}^{L_{n}}\left(L_{n}-v\right)[p(v, t)-n(v, t)] d v .
\end{gathered}
$$

To determine spatiotemporal distributions of concentrations of carriers let us transform the Eqs. (1) to integral form. The integral form of Eqs. (1) Is presented in Appendix. Integral form of the Eqs. (1) after accounting the relation (5) is presented in the Appendix.

Let us solve the system of equations $(1 a)$ by method of averaging of functional correction [11,12]. Framework this method we replace concentrations of charge carriers $n(x, t)$ and $p(x, t)$ on their average values $\alpha_{n 1}$ and $\alpha_{p 1}$, which not yet known now. The replacement gives us possibility to obtain first-order approximations of the concentrations in the following form

$$
\begin{aligned}
& n_{1}(x, t)=\alpha_{n 1}+\left(g(x, t)+\left(\alpha_{p 1}-\alpha_{n 1}\right) \frac{e \alpha_{n 1}}{\varepsilon \varepsilon_{0}} \int_{0}^{t} \int_{x}^{L_{n}} \mu_{n}\left(L_{n}-v\right) d v d \tau-\int_{0}^{t} \int_{L_{n}}^{x} \mu_{n} d v d \tau\left[\frac{\varphi_{k}+U(t)}{L_{p}+L_{n}}-\right.\right. \\
& \left.-e\left(\alpha_{p 1}-\alpha_{n 1}\right) \frac{L_{n}+L_{p}}{2 \varepsilon \varepsilon_{0}}\right] \alpha_{n 1}-\int_{0}^{t} \int_{L_{n}}^{x}(x-v) k_{n p}\left(\alpha_{n 1} \alpha_{p 1}-n_{0} p_{0}\right) d v d \tau+\int_{0}^{t} D_{n} n(x, \tau) d \tau-\alpha_{n 1} \times \\
& \times D_{n 0}(x, t)-\int_{0}^{t} D_{n}\left(L_{n}\right) n_{n}(\tau) d \tau+\int_{0}^{L_{n}}\left(L_{n}-v\right)\left[\alpha_{n 1}-n_{0}(v)\right] d v+\alpha_{n 1} \int_{0}^{t} D_{n}\left(L_{n}\right) d \tau-g\left(L_{n}, t\right)-
\end{aligned}
$$




$$
\begin{aligned}
& -\alpha_{n 1} \int_{0}^{t} D_{n} d \tau+\int_{0}^{x}(x-v) n_{0}(v) d v-\alpha_{n 1} \frac{x^{2}}{2}+\left\{\left[\frac{\varphi_{k}}{L_{p}+L_{n}}-\left(\alpha_{p 1}-\alpha_{n 1}\right)\left(L_{n}+L_{p}\right) \frac{e \alpha_{n 1}}{2 \varepsilon \varepsilon_{0}}\right] \times\right. \\
& \times \int_{0}^{t} \int_{-L_{p}}^{L_{n}} \mu_{n} d v d \tau-\int_{0}^{t} \int_{-L_{p}}^{L_{n}}\left(\alpha_{n 1} \alpha_{p 1}-n_{0} p_{0}\right) k_{n p}\left(L_{p}+v\right) d v d \tau+\frac{e \alpha_{n 1}}{\varepsilon_{0} \varepsilon_{0}} \int_{0}^{t} \int_{-L_{p}}^{L_{n}}\left(L_{n}-v\right) \mu_{n} d v d \tau \times \\
& \times\left(\alpha_{p 1}-\alpha_{n 1}\right)+\alpha_{n 1} D_{n 0}\left(-L_{p}, t\right)-\int_{-L_{p}}^{0}\left(L_{p}+v\right)\left[\alpha_{n 1}-n_{0}(v)\right] d v-\int_{0}^{t} D_{n}\left(L_{n}\right) n_{n}(\tau) d \tau+\int_{0}^{t} n_{p}(\tau) \times \\
& \times D_{n}\left(-L_{p}\right) d \tau+\alpha_{n 1} \int_{0}^{t} D_{n}\left(L_{n}\right) d \tau+g\left(L_{p}, t\right)+\int_{0}^{L_{n}}\left(L_{n}-v\right)\left[\alpha_{n 1}-n_{0}(v)\right] d v-\int_{0}^{t} D_{n}\left(-L_{p}\right) d \tau \times \\
& \left.\times \alpha_{n 1}-g\left(L_{n}, t\right)+g\left(L_{p}, t\right)+\int_{0}^{L_{n}}\left(L_{n}-v\right)\left[\alpha_{n 1}-n_{0}(v)\right] d v-\alpha_{n 1} \int_{0}^{t} D_{n}\left(-L_{p}\right) d \tau-g\left(L_{n}, t\right)\right\} \times \\
& \left.\times \frac{x-L_{n}}{L_{p}+L_{n}}\right) \frac{1}{L^{2}} \\
& p_{1}(x, t)=\alpha_{p 1}+\frac{1}{L^{2}}\left(g(x, t)-\alpha_{p 1} \int_{0}^{x}(x-v) d v+\left(\alpha_{p 1}-\alpha_{n 1}\right) \frac{e \alpha_{p 1}}{\varepsilon \varepsilon_{0}} \int_{0}^{t} \int_{L_{n}}^{x}\left(L_{n}-v\right) \mu_{p} d v d \tau+\right. \\
& +\alpha_{p 1}\left[\frac{\Delta \varphi_{k}+U(t)}{L_{p}+L_{n}}-e\left(\alpha_{p 1}-\alpha_{n 1}\right) \frac{L_{p}+L_{n}}{2 \varepsilon \varepsilon_{0}}\right] \int_{0}^{t} \int_{L_{n}}^{x} \mu_{p} d v d \tau-\int_{0}^{t} \int_{L_{n}}^{x}(x-v) k_{n p}\left[\alpha_{n 1} \alpha_{p 1}-n_{0} p_{0}\right] d v d \tau+ \\
& +\alpha_{p 1} \int_{0}^{t} D_{p} d \tau+\alpha_{p 1} D_{p 0}(x, t)-\int_{0}^{t} D_{p}\left(L_{n}\right) p_{n}(\tau) d \tau+\alpha_{p 1} \frac{L_{n}^{2}}{2}-g\left(L_{n}, t\right)-\int_{0}^{t}\left[D_{p}-D_{p}\left(L_{n}\right)\right] d \tau \times \\
& \times \alpha_{p 1}+\frac{x-L_{n}}{L_{p}+L_{n}}\left\{\left(\alpha_{p 1}-\alpha_{n 1}\right) \frac{\alpha_{p 1} e}{\varepsilon \varepsilon_{0}} \int_{0}^{t} \int_{-L_{p}}^{L_{n}} \mu_{p}\left(L_{n}-v\right) d v d \tau+\alpha_{p 1} \int_{0}^{t} \int_{-L_{p}}^{L_{n}} \mu_{p} d v d \tau\left[\frac{\varphi_{k}+U(t)}{L_{p}+L_{n}}-\left(\alpha_{p 1}-\right.\right.\right. \\
& \left.\left.-\alpha_{n 1}\right) e \frac{L_{p}+L_{n}}{\varepsilon \varepsilon_{0}}\right]+\int_{0}^{t} \int_{-L_{p}}^{L_{n}}\left(L_{p}+v\right)\left(\alpha_{n 1} \alpha_{p 1}-n_{0} p_{0}\right) k_{n p} d v d \tau-g\left(-L_{p}, t\right)+\alpha_{p 1} D_{p 0}\left(-L_{p}, t\right)+ \\
& +\alpha_{p 1} \frac{L_{n}^{2}}{2}-\int_{0}^{t} D_{p}\left(L_{n}\right) p_{n}(\tau) d \tau+\int_{0}^{t} D_{p}\left(-L_{p}\right) p_{p}(\tau) d \tau-g\left(L_{n}, t\right)+\int_{0}^{t}\left[D_{p}\left(L_{n}\right)-D_{p}\left(-L_{p}\right)\right] d \tau \times \\
& \left.\left.\times \alpha_{p 1}-\frac{\alpha_{p 1}}{2} L_{p}^{2}\right\}\right)
\end{aligned}
$$

Let us integrate Eq.(6) in limits from $-L_{p}$ to $L_{n}$ at coordinate and from 0 to $\Theta$ at time. After the integration we obtain system of equations for average values of charge carriers concentrations $\alpha_{n 1}$ и $\alpha_{p 1}$. The system is bulky. Therefore is presented in Appendix. The second-order approximations of charge carrier's concentrations could be calculated by standard iterative procedure [11,12]. Framework the iterative procedure the replacement $n(x, t) \rightarrow \alpha_{n 2}+n_{1}(x, t)$ and $p(x, t) \rightarrow \alpha_{p 2}+p_{1}(x, t)$ should be done, where $\alpha_{n 2}$ and $\alpha_{p 2}$ are the average values of the second-order approximations of concentrations of charge carriers $n_{2}(x, t)$ and $p_{2}(x, t)$. After the replacement in the Eqs. $(1 a)$ we obtain the second-order approximations concentrations of charge carriers 


$$
\begin{aligned}
& n_{2}(x, t)=\alpha_{n 2}+n_{1}(x, t)+\frac{1}{L^{2}}\left\{g(x, t)+\left(\alpha_{p 2}-\alpha_{n 2}\right) \frac{\alpha_{n 2} e}{\varepsilon \varepsilon_{0}} \int_{0}^{t} \int_{x}^{L_{n}} \mu_{n}\left(L_{n}-v\right) d v d \tau+\widetilde{A}_{n 01111}(x, t) \times\right. \\
& \quad \times \frac{\alpha_{n 2} e}{\varepsilon \varepsilon_{0}}-\int_{0}^{t} \int_{L_{n}}^{x} \mu_{n}\left[\alpha_{n 2}+n_{1}(v, \tau)\right] d v d \tau+\frac{e}{\varepsilon \varepsilon_{0}} \widetilde{A}_{n 111111}(x, t)+e \int_{0}^{t} \int_{x}^{L_{n}} \mu_{n}\left(L_{n}-v\right) n_{1}(v, \tau) d v d \tau \times \\
& \quad \times \frac{\alpha_{p 2}-\alpha_{n 2}}{\varepsilon \varepsilon_{0}}-\int_{0}^{x}(x-v)\left[\alpha_{n 2}+n_{1}(v, t)\right] d v\left[U(t)-\frac{e L_{p}}{\varepsilon \varepsilon_{0}}\left(\alpha_{p 2}-\alpha_{n 2}\right)\left(L_{n}-L_{p}\right)-\frac{e}{\varepsilon \varepsilon_{0}} \widetilde{B}_{1111}+\right. \\
& \left.+\varphi_{k}\right] \frac{1}{L_{p}+L_{n}}-\int_{0}^{t} \int_{L_{n}}^{x}(x-v) k_{n p}\left\{\left[\alpha_{n 2}+n_{1}(v, \tau)\right]\left[\alpha_{p 2}+p_{1}(v, \tau)\right]-n_{0} p_{0}\right\} d v d \tau+\int_{0}^{x} n_{0}(v)(x-
\end{aligned}
$$$$
-v) d v+\alpha_{n 2} \frac{L_{n}^{2}}{2}+\widetilde{G}_{n 11}\left(L_{n}, t\right)-\int_{0}^{t} D_{n}\left(L_{n}\right) n_{n}(\tau) d \tau-\widetilde{D}_{n 11}(x, t)-\alpha_{n 2} \widetilde{D}_{n 10}(x, t)-\alpha_{n 2} \widetilde{F}_{n 10}(x, t)-
$$$$
-\widetilde{F}_{n 11}(x, t)-g\left(L_{n}, t\right)+\frac{x-L_{n}}{L_{p}+L_{n}}\left(\frac{e}{\varepsilon \varepsilon_{0}} \int_{0}^{t} \int_{-L_{p}}^{L_{n}}\left[\alpha_{n 2}+n_{1}(v, \tau)\right]\left[\alpha_{p 2}+p_{1}(v, \tau)-\alpha_{n 2}-n_{1}(v, \tau)\right] \times\right.
$$$$
\times \mu_{n}\left(L_{n}-v\right) d v d \tau+g\left(-L_{p}, t\right)+\frac{1}{L_{p}+L_{n}}\left[\varphi_{k}+U(t)-\frac{e L_{p}}{\varepsilon \varepsilon_{0}}\left(\alpha_{p 2}-\alpha_{n 2}\right)\left(L_{n}-L_{p}\right)-\frac{e}{\varepsilon \varepsilon_{0}} \times\right.
$$$$
\left.\times \widetilde{B}_{1111}\right] \int_{0}^{t} \int_{-L_{p}}^{L_{n}} \mu_{n}\left[\alpha_{n 2}+n_{1}(v, \tau)\right] d v d \tau-\int_{0}^{t} D_{n}\left(L_{n}\right) n_{n}(\tau) d \tau-\int_{0}^{t} \int_{-L_{p}}^{L_{n}}\left\{\left[\alpha_{n 2}+n_{1}(v, \tau)\right]\left[\alpha_{p 2}+p_{1}(v, \tau)\right]-\right.
$$$$
\left.-n_{0} p_{0}\right\} k_{n p}\left(L_{p}+v\right) d v d \tau-\frac{3}{2} \alpha_{n 2} L_{p}^{2}-\widetilde{G}_{n 11}\left(-L_{p}, t\right)-g\left(L_{n}, t\right)+\int_{0}^{t} D_{n}\left(-L_{p}\right) n_{p}(\tau) d \tau+\frac{\alpha_{n 2}}{2} \times
$$

$$
\left.\left.\times L_{n}^{2}+\widetilde{G}_{n 11}\left(L_{n}, t\right)-\alpha_{n 2} \widetilde{D}_{n 10}\left(-L_{p}, t\right)+\widetilde{D}_{n 11}\left(-L_{p}, t\right)-\alpha_{n 2} \widetilde{F}_{n 10}\left(-L_{p}, t\right)-\widetilde{F}_{n 11}\left(-L_{p}, t\right)\right)\right\},
$$

$p_{2}(x, t)=\alpha_{p 2}+p_{1}(x, t)+\frac{1}{L^{2}}\left\{g(x, t)+\left(\alpha_{p 2}-\alpha_{n 2}\right) \frac{\alpha_{p 2} e}{\varepsilon \varepsilon_{0}} \int_{0}^{t} \int_{x}^{L_{n}} \mu_{p}\left(L_{n}-v\right) d v d \tau+\widetilde{A}_{p 011111}(x, t) \times\right.$ $\times \frac{\alpha_{p 2} e}{\varepsilon \varepsilon_{0}}-\int_{0}^{t} \int_{L_{n}}^{x} \mu_{n}\left[\alpha_{p 2}+p_{1}(v, \tau)\right] d v d \tau+\frac{e}{\varepsilon \varepsilon_{0}} \widetilde{A}_{p 111111}(x, t)+e \int_{0}^{t} \int_{x}^{L_{n}} \mu_{p}\left(L_{n}-v\right) p_{1}(v, \tau) d v d \tau \times$ $\times \frac{\alpha_{p 2}-\alpha_{n 2}}{\varepsilon \varepsilon_{0}}-\int_{0}^{x}(x-v)\left[\alpha_{p 2}+p_{1}(v, t)\right] d v\left[U(t)-\frac{e L_{p}}{\varepsilon \varepsilon_{0}}\left(\alpha_{p 2}-\alpha_{n 2}\right)\left(L_{n}-L_{p}\right)-\frac{e}{\varepsilon \varepsilon_{0}} \widetilde{B}_{1111}+\right.$ $\left.+\varphi_{k}\right] \frac{1}{L_{p}+L_{n}}-\int_{0}^{t} \int_{L_{n}}^{x}(x-v) k_{n p}\left\{\left[\alpha_{n 2}+n_{1}(v, \tau)\right]\left[\alpha_{p 2}+p_{1}(v, \tau)\right]-n_{0} p_{0}\right\} d v d \tau+\int_{0}^{x} p_{0}(v)(x-$ $-v) d v+\alpha_{p 2} \frac{L_{n}^{2}}{2}+\widetilde{G}_{p 11}\left(L_{n}, t\right)-\int_{0}^{t} D_{p}\left(L_{n}\right) p_{p}(\tau) d \tau-\widetilde{D}_{p 11}(x, t)-\alpha_{p 2} \widetilde{D}_{p 10}(x, t)-\alpha_{p 2} \widetilde{F}_{p 10}(x, t)-$ 


$$
\begin{aligned}
& -\widetilde{F}_{p 11}(x, t)-g\left(L_{n}, t\right)+\frac{x-L_{n}}{L_{p}+L_{n}}\left(\frac{e}{\varepsilon \varepsilon_{0}} \int_{0}^{t} \int_{-L_{p}}^{L_{n}}\left[\alpha_{n 2}+n_{1}(v, \tau)\right]\left[\alpha_{p 2}+p_{1}(v, \tau)-\alpha_{n 2}-n_{1}(v, \tau)\right] \times\right. \\
& \times \mu_{p}\left(L_{n}-v\right) d v d \tau+g\left(-L_{p}, t\right)+\frac{1}{L_{p}+L_{n}}\left[\varphi_{k}+U(t)-\frac{e L_{p}}{\varepsilon \varepsilon_{0}}\left(\alpha_{p 2}-\alpha_{n 2}\right)\left(L_{n}-L_{p}\right)-\frac{e}{\varepsilon \varepsilon_{0}} \times\right. \\
& \left.\times \widetilde{B}_{1111}\right]_{0}^{t} \int_{0-L_{p}}^{L_{n}} \mu_{p}\left[\alpha_{p 2}+p_{1}(v, \tau)\right] d v d \tau-\int_{0}^{t} D_{p}\left(L_{n}\right) p_{p}(\tau) d \tau-\int_{0-L_{p}}^{t} \int_{L_{p}}\left\{\left[\alpha_{n 2}+n_{1}(v, \tau)\right]\left[\alpha_{p 2}+p_{1}(v, \tau)\right]-\right. \\
& \left.-n_{0} p_{0}\right\} k_{n p}\left(L_{p}+v\right) d v d \tau-\frac{3}{2} \alpha_{p 2} L_{p}^{2}-\widetilde{G}_{p 11}\left(-L_{p}, t\right)-g\left(L_{n}, t\right)+\int_{0}^{t} D_{p}\left(-L_{p}\right) p_{p}(\tau) d \tau+\frac{\alpha_{p 2}}{2} \times \\
& \left.\left.\quad \times L_{n}^{2}+\widetilde{G}_{p 11}\left(L_{n}, t\right)-\alpha_{p 2} \widetilde{D}_{p 10}\left(-L_{p}, t\right)+\widetilde{D}_{p 11}\left(-L_{p}, t\right)-\alpha_{p 2} \widetilde{F}_{p 10}\left(-L_{p}, t\right)-\widetilde{F}_{p 11}\left(-L_{p}, t\right)\right)\right\},
\end{aligned}
$$

where

$$
\begin{array}{r}
\widetilde{A}_{\rho i j k l m n}(x, t)=\int_{0}^{t} \int_{x}^{L_{n}} \mu_{\rho}\left(L_{n}-v\right) \rho_{j}^{i}(v, \tau)\left[p_{l}^{k}(v, \tau)-n_{n}^{m}(v, \tau)\right] d v d \tau, \widetilde{B}_{i j k l}=\int_{-L_{p}}^{L_{n}}\left(L_{n}-v\right) \times \\
\times\left[p_{j}^{i}(v, t)-n_{l}^{k}(v, t)\right] d v, \widetilde{C}_{i j}(x, t)=\int_{0}^{t} \int_{L_{n}}^{x}(x-v) k_{n p}\left[n^{i}(v, \tau) p^{j}(v, \tau)-n_{0} p_{0}\right] d v d \tau, \widetilde{D}_{\rho i j}(x, t)= \\
=\int_{0}^{t} \int_{L_{n}}^{x} \mu_{\rho} \rho_{j}^{i}(v, \tau) \frac{\partial \varphi_{h}(v, \tau)}{\partial v} d v d \tau, \\
\widetilde{G}_{\rho i j}(x, t)=\int_{0}^{x}\left(L_{n}-v\right)\left[\rho_{j}^{i}(v, t)-\rho_{0}(v)\right] d v \\
\quad=\int_{0}^{t} \int_{L_{n}}^{x} \rho_{j}^{i}(v, \tau) \frac{\partial \widetilde{F}_{\rho i j}(x, t)=}{\partial v} d v d \tau .
\end{array}
$$

Parameters $\alpha_{n 2}$ and $\alpha_{p 2}$ have been determined framework standard procedure of method of averaging of function corrections, i.e.

$$
\alpha_{\rho 2}=\frac{1}{\Theta L} \int_{0}^{\Theta} \int_{0}^{L}\left[\rho_{2}(x, t)-\rho_{1}(x, t)\right] d x d t
$$

Here $\rho$ are the concentrations of electrons and holes in such relations, which are the same with the exception of the concentrations; $\Theta$ is time of observation on dynamics of carriers. Substitution of the first- and the second-order approximations of carriers' concentrations in the relation (7) give us possibility to obtain system of equations for parameters $\alpha_{n 2}$ and $\alpha_{p 2}$. The system is presented in the Appendix. Solution $\alpha_{n 2}$ and $\alpha_{p 2}$ of the system is

$$
\alpha_{p 2}=\sqrt[3]{\frac{p^{3}}{27}+\frac{q^{2}}{4}}-\frac{q}{2}-\sqrt[3]{\frac{p^{3}}{27}+\frac{q^{2}}{4}+\frac{q}{2}}-\frac{b_{2} b_{8}+b_{1} b_{9}}{3\left(b_{2} b_{6}+b_{1} b_{7}\right)}, \alpha_{n 2}=\frac{b_{1} \alpha_{n 2}^{2}-b_{4} \alpha_{p 2}-b_{5}}{b_{2} \alpha_{p 2}+b_{3}},
$$

where 


$$
\begin{gathered}
q=\frac{2}{27}\left(\frac{b_{2} b_{8}+b_{1} b_{9}}{b_{2} b_{6}+b_{1} b_{7}}\right)^{3}-\left(b_{2} b_{8}+b_{1} b_{9}\right) \frac{\left(b_{3} b_{8}-b_{7} b_{4}-b_{5} b_{7}-b_{4} b_{9}+b_{2} b_{10}\right)}{3\left(b_{2} b_{6}+b_{1} b_{7}\right)^{2}}+\frac{b_{3} b_{10}-b_{5} b_{9}}{b_{2} b_{6}+b_{1} b_{7}} \\
p=\frac{b_{3} b_{8}-b_{7} b_{4}-b_{5} b_{7}-b_{4} b_{9}+b_{2} b_{10}}{b_{2} b_{6}+b_{1} b_{7}}-\frac{1}{3}\left(\frac{b_{2} b_{8}+b_{1} b_{9}}{b_{2} b_{6}+b_{1} b_{7}}\right)^{2}
\end{gathered}
$$

Relations for the parameters $b_{i}$ are presented in the Appendix.

Analysis of spatiotemporal distributions of charge carriers has been done analytically by using their second-order approximation and has been amended numerically.

\section{Discussion}

Let us analyzed solution of the system of equations $(1 a)$ and match the solution with experimental results. Usually in the relative works one can analyzed current-voltage characteristic (CVC). The following relation could determine density of common current $J \mathrm{~A} \cdot \mathrm{cm}^{-2}$ in any point of in homogenous sample at any moment of time

$$
J=e\left[D_{n} \frac{\partial n(x, t)}{\partial x}-D_{p} \frac{\partial p(x, t)}{\partial x}\right]-e\left[\mu_{n} n(x, t)+\mu_{p} p(x, t)\right] \frac{\partial\left[\varphi(x, t)+\varphi_{h}(x, t)\right]}{\partial x} .
$$

With account Eq.(5) the Eq.(8) could be written as

$$
\begin{gathered}
J=e\left[D_{n} \frac{\partial n(x, t)}{\partial x}-D_{p} \frac{\partial p(x, t)}{\partial x}\right]-e\left[\mu_{n} n(x, t)+\mu_{p} p(x, t)\right] \frac{\partial \varphi_{h}(x, t)}{\partial x}-e\left[\mu_{n} n(x, t)+\right. \\
\left.+\mu_{p} p(x, t)\right]\left(\varphi_{k}+U(t)+\frac{x-L_{n}}{L_{p}+L_{n}}\left\{\varphi_{k}+U(t)-\frac{e}{\varepsilon \varepsilon_{0}} \int_{-L_{p}}^{L_{n}}\left(L_{n}-v\right)[p(v, t)-n(v, t)] d v\right\}-\right. \\
\left.-\frac{e}{\varepsilon \varepsilon_{0}} \int_{x}^{L_{n}}\left(L_{n}-v\right)[p(v, t)-n(v, t)] d v\right)
\end{gathered}
$$

Fig. 3 shows experimental CVC of diffusion-junction rectifier in comparison with two calculated CVCs. One of the CVCs has been calculated by using considered in this paper approach. The second one is "ideal" CVC [4]. The "ideal" CVC has been calculated by using diffusion and drift terms in Eqs.(1), homogenous sample and constant in time parameters.

\section{Conclusion}

In this paper we consider an approach to calculate spatiotemporal distributions of charge carriers concentrations in a $p$ - $n$-junction. We compare calculated current-voltage characteristics with experimental one. In this paper we used two approaches to model current-voltage characteristics. First of them is introduced in this paper approach. The second one is more simple approach from literature. As a result of calculation we show, that accuracy of calculation of current-voltage characteristics increases. 


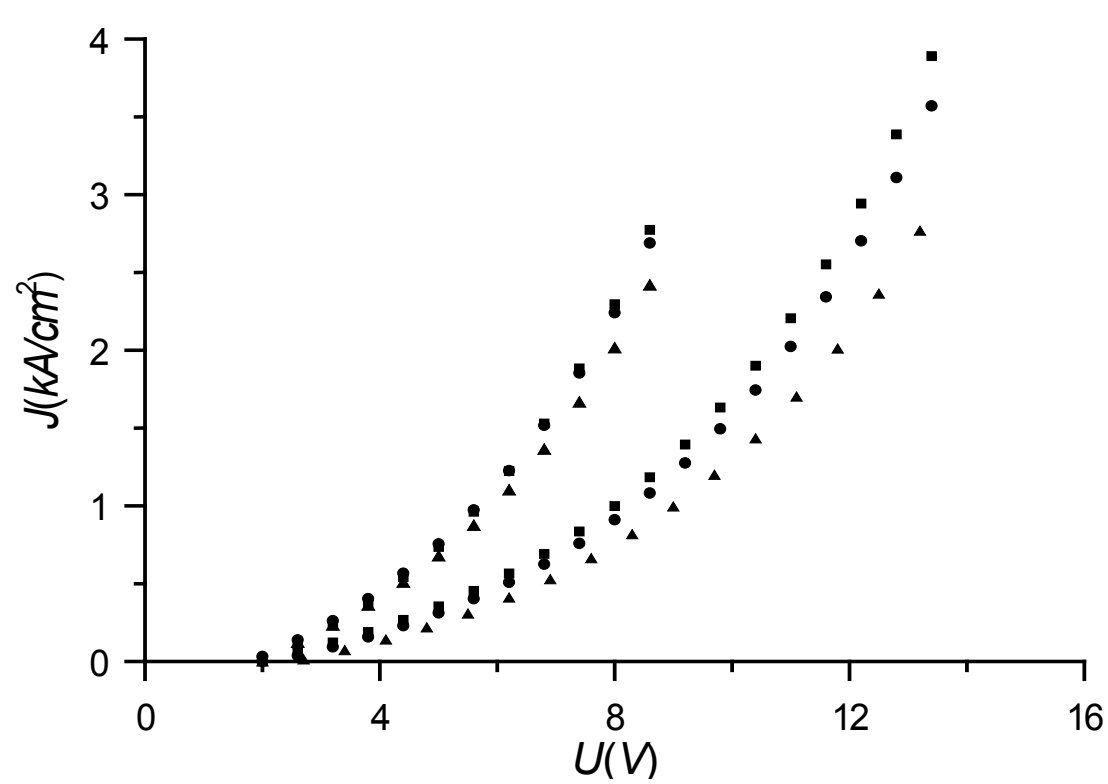

Figure 3a. Direct branch of CVC of diffusion-junction rectifier. Four squares are experimental data from [13]; circles is CVC, calculated by using approach from this paper; triangles is CVC, calculated by using model of "ideal" CVC [4].

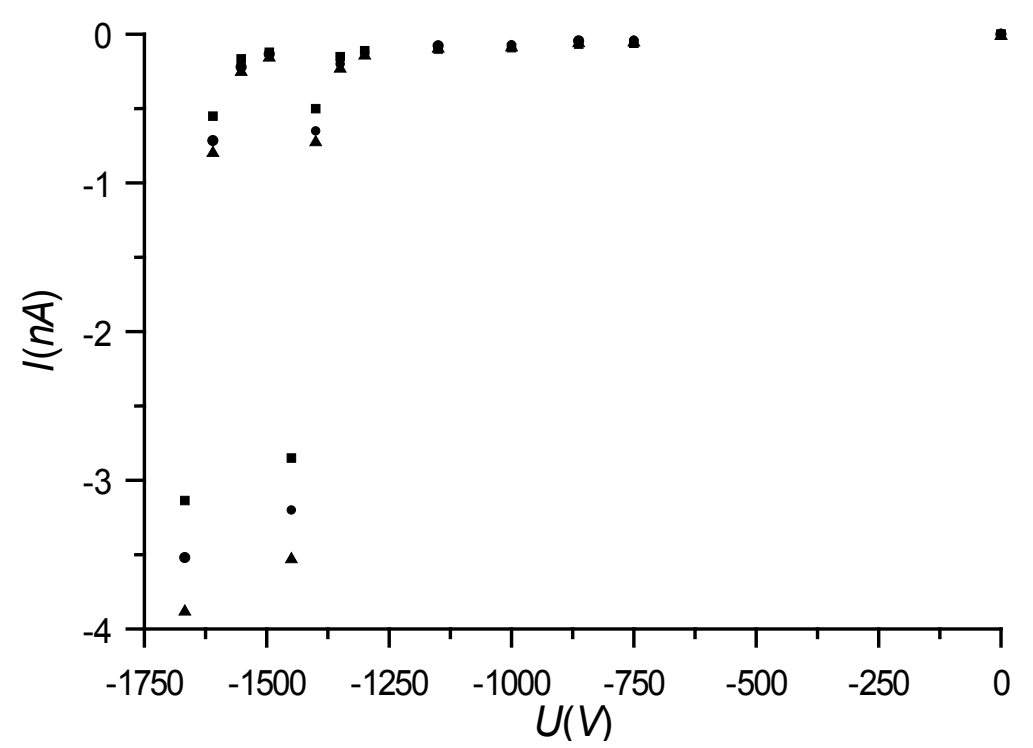

Figure 3b. Reverse branch of CVC of diffusion-junction rectifier. Four squares are experimental data from [13]; circles is CVC, calculated by using approach from this paper; triangles is CVC, calculated by using model of "ideal" CVC [4]Appendix

Integral form of Eqs. (1)

$$
\begin{gathered}
n(x, t)=n(x, t)+\frac{1}{L^{2}}\left(g(x, t)+\frac{e}{\varepsilon \varepsilon_{0}} A_{n 111}(x, t)-\int_{0}^{x}(x-v) n(v, t) d v-\frac{1}{L_{p}+L_{n}}\left\{\varphi_{k}-\right.\right. \\
\left.-\frac{e}{\varepsilon \varepsilon_{0}} B_{11}+U(t)\right\} \int_{0}^{t} \int_{0}^{x} \mu_{n} n(v, \tau) d v d \tau+\int_{0}^{x}(x-v) n_{0}(v) d v-C_{11}(x, t)-D_{n 1}(x, t)-F_{n 1}(x, t)+ \\
+\int_{0}^{t} D_{n} n(x, \tau) d \tau-\int_{0}^{t} D_{n}\left(L_{n}\right) n_{n}(\tau) d \tau+G_{n 1}\left(L_{n}, t\right)-g\left(L_{n}, t\right)+\frac{x-L_{n}}{L_{p}+L_{n}}\left\{\frac { 1 } { L _ { p } + L _ { n } } \left[\varphi_{k}+\right.\right.
\end{gathered}
$$




$$
\begin{aligned}
& \left.\left.+U(t)-\frac{e}{\varepsilon \varepsilon_{0}} A_{011}\left(-L_{p}, t\right)\right]\right]_{0}^{t} \int_{-L_{p}}^{L_{n}} \mu_{n} n(v, \tau) d v d \tau+\frac{e}{\varepsilon \varepsilon_{0}} A_{111}\left(-L_{p}, t\right)-\int_{0}^{t} n_{n}(\tau) D_{n}\left(L_{n}\right) d \tau+ \\
& +g\left(-L_{p}, t\right)+C_{11}\left(-L_{p}, t\right)+G_{n 1}\left(-L_{p}, t\right)+D_{n 1}-F_{n 1}\left(-L_{p}\right)+\int_{0}^{t} D_{n}\left(-L_{p}\right) n_{p}(\tau) d \tau- \\
& \left.\left.-g\left(L_{n}, t\right)+G_{n 1}\left(L_{n}, t\right)\right\}\right) \\
& p(x, t)=p(x, t)+\frac{1}{L^{2}}\left\{g(x, t)-\int_{0}^{x}(x-v) p(v, t) d v+D_{p 1}(x, t)+\frac{e}{\varepsilon \varepsilon_{0}} A_{p 111}(x, t)+\frac{1}{L_{p}+L_{n}} \times\right. \\
& \times\left[U(t)-\frac{e}{\varepsilon \varepsilon_{0}} A_{p 011}\left(-L_{p}, t\right)+\varphi_{k}\right] \int_{0}^{t} \int_{L_{n}}^{x} \mu_{p} p(v, \tau) d v d \tau+\int_{0}^{t} D_{p} p(x, \tau) d \tau-\int_{0}^{t} D_{p}\left(L_{n}\right) p_{n}(\tau) d \tau- \\
& -C_{11}(x, t)-F_{p 1}+\int_{0}^{L_{n}}\left(L_{n}-v\right) p(v, t) d v-g\left(L_{n}, t\right)+\left(\frac { 1 } { L _ { p } + L _ { n } } \left[U(t)-A_{011}\left(-L_{p}, x\right) \times\right.\right. \\
& \left.\times \frac{e}{\varepsilon \varepsilon_{0}}+\varphi_{k}\right] \int_{0}^{t} \int_{-L_{p}}^{L_{n}} \mu_{p} p(v, \tau) d v d \tau+D_{p 1}+\int_{0}^{L_{n}}\left(L_{n}-v\right) p(v, t) d v+\frac{e}{\varepsilon \varepsilon_{0}} A_{p 111}+C_{11}\left(-L_{p}, t\right)+ \\
& \left.\left.+g\left(-L_{p}, t\right)-F_{p 1}\left(-L_{p}, t\right)-g\left(L_{n}, t\right)+\int_{0}^{t} D_{p}\left(-L_{p}\right) p_{p}(\tau) d \tau\right) \frac{x-L_{n}}{L_{p}+L_{n}}\right\},
\end{aligned}
$$

where

$$
\begin{aligned}
& g(x, t)=\int_{0}^{t} \int_{0}^{x}(x-v) G d v d \tau \quad A_{\rho i j k}(x, t)=\int_{0}^{t} \int_{x}^{L_{n}} \mu_{\rho}\left(L_{n}-v\right) \rho^{i}(v, \tau)\left[p^{j}(v, \tau)-n^{k}(v, \tau)\right] d v d \tau \\
& B_{i j}=\int_{-L_{p}}^{L_{n}}\left(L_{n}-v\right)\left[p^{i}(v, t)-n^{j}(v, t)\right] d v, C_{i j}(x, t)=\int_{0}^{t} \int_{L_{n}}^{x}(x-v) k_{n p}\left[n^{i}(v, \tau) p^{j}(v, \tau)-n_{0} p_{0}\right] d v d \tau, \\
& D_{\rho i}(x, t)=\int_{0}^{t} \int_{L_{n}}^{x} \mu_{\rho} \rho^{i}(v, \tau) \frac{\partial \varphi_{h}(v, \tau)}{\partial v} d v d \tau, \quad G_{\rho i}(x, t)=\int_{0}^{x}\left(L_{n}-v\right)\left[\rho^{i}(v, t)-\rho_{0}(v)\right] d v, \\
& F_{\rho i}(x, t)=\int_{0}^{t} \int_{L_{n}}^{x} \rho^{i}(v, \tau) \frac{\partial D_{\rho}}{\partial v} d v d \tau
\end{aligned}
$$

System of equations for parameters $\alpha_{n 1}$ and $\alpha_{p 1}$

$$
\frac{1}{2} \hat{G}_{12}-\frac{1}{2} \hat{G}_{22}-\alpha_{n 1}\left(L_{n}^{3}+L_{p}^{3}\right) \frac{\Theta}{6}+\frac{e \alpha_{n 1}}{\varepsilon \varepsilon_{0}}\left(\alpha_{p 1}-\alpha_{n 1}\right)\left[\left(L_{n}+L_{p}\right) H_{n 1}-H_{n 2}-\int_{0}^{\Theta}(\Theta-t) \int_{-L_{p}}^{0} \mu_{n} \times\right.
$$




$$
\begin{aligned}
& \left.\times\left(L_{n}-x\right)\left(L_{p}+x\right) d x d \tau\right]-\frac{1}{2} \int_{0}^{\Theta}(\Theta-t) \int_{-L_{p}}^{L_{n}}\left(L_{p}+x\right)^{2} k_{n p}\left(\alpha_{n 1} \alpha_{p 1}-n_{0} p_{0}\right) d x d t-\frac{\left(L_{p}+L_{n}\right)^{2}}{2} \times \\
& \times \int_{0}^{\Theta}(\Theta-t) A_{n}(t) \int_{0}^{L_{n}}\left(L_{n}-x\right) \mu_{n} d x d t d t+\alpha \int_{n 1}^{\Theta}(\Theta-t) \int_{0}^{L_{n}} D_{n} d x d \tau-\alpha_{n 1} \hat{B}_{n}+\Theta\left(L_{n}+L_{p}\right) \times \\
& \times L_{n}^{2} \frac{\alpha_{n 1}}{2}-\Theta\left(L_{n}+L_{p}\right) I_{n 1}-\left(L_{n}+L_{p}\right) \int_{0}^{\Theta}(\Theta-t) n_{n}(t) D_{n}\left(L_{n}\right) d t-\alpha_{n 1} \int_{0}^{\Theta}(\Theta-t) \int_{-L_{p}}^{L_{n}} D_{n} d x d t+ \\
& \times \int_{0}^{L_{n}}\left(L_{n}-x\right)\left[\alpha_{n 1}-n_{0}(x)\right] d x-\left(L_{n}+L_{p}\right) \int_{0}^{\Theta}(\Theta-t) n_{n}(t) D_{n}\left(L_{n}\right) d t-\alpha_{n 1} \int_{0}^{\Theta}(\Theta-t) \int_{-L_{p}}^{L_{n}} D_{n} d x d t+ \\
& +\frac{1}{2} I_{n 2}+\alpha_{n 1}\left(L_{n}+L_{p}\right) \int_{0}^{\Theta}(\Theta-t) D_{n}\left(L_{n}\right) d t-\left(L_{n}+L_{p}\right) \hat{G}_{11}+\frac{1}{2} J_{n 2}-\frac{\left(L_{n}+L_{p}\right)^{2}}{2\left(L_{p}+L_{n}\right)}\left\{\int_{0}^{\Theta}(\Theta-t) \times\right. \\
& \times A_{n}(t) \int_{-L_{p}}^{L_{n}} \mu_{n} d x d t-\int_{0}^{\Theta}(\Theta-t) \int_{-L_{p}}^{L_{n}}\left(\alpha_{n 1} \alpha_{p 1}-n_{0} p_{0}\right)\left(L_{p}+x\right) k_{n p} d x d t+\int_{0}^{\Theta} \int_{-L_{p}}^{L_{n}} \mu_{n}\left(L_{n}-x\right) d x \times \\
& \times(\Theta-t) d t+\left(\alpha_{p 1}-\alpha_{n 1}\right) \frac{\alpha_{n 1} e}{\varepsilon_{0} \varepsilon}+\alpha_{n 1} \int_{0}^{\Theta}(\Theta-t) D_{n}\left(L_{n}\right) d t-\int_{0}^{\Theta}(\Theta-t) D_{n}\left(L_{n}\right) n_{n}(t) d t+ \\
& +\hat{G}_{21}+\alpha_{n 1} \hat{B}_{n}-\alpha_{n 1} L_{p}^{2} \frac{\Theta}{2}+\Theta J_{n 1}+\alpha_{n 1} L_{n}^{2} \frac{\Theta}{2}-\Theta I_{n 1}-\alpha_{n 1} \int_{0}^{\Theta}(\Theta-t) D_{n}\left(-L_{p}\right) d t-\hat{G}_{11}+ \\
& \left.+\int_{0}^{\Theta}(\Theta-t) D_{n}\left(-L_{p}\right) n_{p}(t) d t\right\}=0 \\
& \frac{1}{2} \hat{G}_{12}+\frac{1}{2} \hat{G}_{22}-\alpha_{p 1} L_{n}^{3} \frac{\Theta}{6}-\alpha_{p 1} L_{p}^{3} \frac{\Theta}{6}+\alpha_{p 1} \int_{0}^{\Theta}(\Theta-t) A_{p}(t) \int_{0}^{L_{n}}\left(L_{n}-x\right) \mu_{p} d x d t-\int_{0}^{\Theta}(\Theta-t) \times \\
& \times A_{p}(t) \int_{-L_{p}}^{0}\left(L_{p}+x\right) \mu_{p} d x d t+\alpha \int_{p 1}^{\Theta}(\Theta-t) \int_{0}^{L_{n}} D_{-L_{p}} d x d t+\int_{0}^{\Theta}(\Theta-t) \int_{-L_{p}}^{L_{n}}\left(L_{n}-x\right) \mu_{p} d x d t \times \\
& \times\left(\alpha_{p 1}-\alpha_{n 1}\right) \frac{e \alpha_{p 1}}{\varepsilon \varepsilon_{0}}-\frac{1}{2} \int_{0}^{\Theta}(\Theta-t) \int_{0}^{L_{n}}\left(L_{n}-x\right)^{2} k_{n p}\left(\alpha_{n 1} \alpha_{p 1}-n_{0} p_{0}\right) d x d t+\frac{1}{2} \int_{0}^{\Theta}(\Theta-t) \times \\
& \times \int_{-L_{p}}^{0}\left(L_{n}+x\right)^{2} k_{n p}\left(\alpha_{n 1} \alpha_{p 1}-n_{0} p_{0}\right) d x d t+\left(L_{n}+L_{p}\right) \int_{0}^{\Theta}(\Theta-t) D_{p}\left(L_{n}\right) p_{n}(t) d t+\alpha_{p 1} \hat{B}_{p 10}- \\
& -\alpha_{p 1} \int_{0}^{\Theta}(\Theta-t) \int_{-L_{p}}^{L_{n}}\left[D_{p}-D_{p}\left(L_{n}\right)\right] d x d t+\Theta \alpha_{p 1}\left(L_{n}+L_{p}\right) \frac{L_{n}^{2}}{2}-\left(L_{n}+L_{p}\right) \hat{G}_{11}-\frac{\left(L_{p}+L_{n}\right)^{2}}{2} \times \\
& \times\left\{A_{\rho}(t) \int_{0}^{\Theta}(\Theta-t) \int_{-L_{p}}^{L_{n}} \mu_{p} d x d t+\left(\alpha_{p 1}-\alpha_{n 1}\right) \frac{\alpha_{p 1} e}{\varepsilon \varepsilon_{0}} \int_{0}^{\Theta}(\Theta-t) \int_{-L_{p}}^{L_{n}} \mu_{p}\left(L_{n}-x\right) d x d t+\alpha_{p 1} \Theta \frac{L_{n}^{2}}{2} \times\right.
\end{aligned}
$$




$$
\begin{array}{r}
\times\left(L_{n}+L_{p}\right)+\hat{G}_{21}+\alpha_{p 1} \int_{0}^{\Theta}(\Theta-t)\left[D_{p}\left(L_{n}\right)-D_{p}\left(-L_{p}\right)\right] d t+\int_{0}^{\Theta}(\Theta-t) \int_{-L_{p}}^{L_{n}}\left(\alpha_{n 1} \alpha_{p 1}-n_{0} p_{0}\right) \times \\
\times k_{n p}\left(L_{p}+x\right) d x d t-\int_{0}^{\Theta}(\Theta-t) p_{n}(t) D_{p}\left(L_{n}\right) d t+\int_{0}^{\Theta}(\Theta-t) D_{p}\left(-L_{p}\right) p_{p}(t) d t-\frac{\alpha_{p 1} \Theta}{2} L_{p}^{2}- \\
\left.-\hat{G}_{11}+\alpha_{p 1} \hat{B}_{p}\right\}=0,
\end{array}
$$

where

$$
\hat{G}_{1 i}=\int_{0}^{\Theta}(\Theta-t) \int_{0}^{L_{n}}\left(L_{n}-x\right)^{i} G d x d t, \hat{G}_{2 i}=\int_{0}^{\Theta}(\Theta-t) \int_{-L_{p}}^{0}\left(L_{p}+x\right)^{i} G d x d t, \quad H_{\rho i}=\int_{0}^{\Theta}(\Theta-t) \times
$$

$\times \int_{0}^{L_{n}} \mu_{\rho}\left(L_{n}-x\right)^{i} d x d \tau, A_{\rho}(t)=\frac{\varphi_{k}+U(t)}{L_{p}+L_{n}}-\alpha_{\rho 1} e\left(\alpha_{p 1}-\alpha_{n 1}\right) \frac{L_{n}+L_{p}}{2 \varepsilon \varepsilon_{0}}, \hat{B}_{\rho i j}=\int_{0}^{\Theta}(\Theta-t) \int_{-L_{p}}^{L_{n}} \mu_{\rho} \times$

$$
\times\left(L_{p}+x\right) \rho_{i}^{j}(x, t) \frac{\partial \varphi_{h}(x, t)}{\partial x} d x d t, \quad I_{\rho i}=\int_{0}^{\Theta} \int_{0}^{L_{n}} \rho_{0}(x)\left(L_{n}-x\right)^{i} d x d t \quad J_{\rho i}=\int_{0}^{\Theta} \int_{0}^{L_{n}}\left(L_{p}+x\right)^{i} \times
$$

$\times \rho_{0}(x) d x d t$

System of equations for parameters $\alpha_{n 2}$ and $\alpha_{p 2}$ could be written as

$$
\begin{aligned}
& \frac{1}{2} \hat{G}_{12}+\frac{e}{\varepsilon \varepsilon_{0}} \int_{0}^{\Theta}(\Theta-t) \int_{-L_{p}}^{L_{n}}\left(L_{p}+x\right)\left(L_{n}-x\right)\left[\alpha_{n 2}+n_{1}(x, t)\right]\left[\alpha_{p 2}+p_{1}(x, t)-\alpha_{n 2}-n_{1}(x, t)\right] \times \\
& \times \mu_{n} d x d t-\frac{1}{2} \int_{0}^{\Theta} \int_{0}^{L_{n}}\left(L_{n}-x\right)\left[\alpha_{n 2}+n_{1}(x, t)\right] d x d t-\frac{1}{2} \int_{0}^{\Theta} \int_{-L_{p}}^{0}\left(L_{p}+x\right)\left[\alpha_{n 2}+n_{1}(x, t)\right] d x d t- \\
& -\int_{0}^{\Theta}\left\{\varphi_{k}+U(t)-\frac{e}{\varepsilon \varepsilon_{0}} \int_{-L_{p}}^{L_{n}}\left(L_{n}-v\right)\left[\alpha_{p 2}+p_{1}(v, t)-\alpha_{n 2}-n_{1}(v, t)\right] d v\right\} \int_{-L_{p}}^{L_{n}}\left[\alpha_{n 2}+n_{1}(x, \tau)\right] \times \\
& \quad \times \mu_{n}\left(L_{p}+x\right) d x d \tau d t \frac{1}{L_{p}+L_{n}-\int_{L_{p}}^{L_{n}} \mu_{n}\left(L_{p}+x\right)\left[\alpha_{n 2}+n_{1}(x, \tau)\right] d x d \tau d t+\frac{1}{2} \int_{0}^{\Theta}(\Theta-t) \times} \\
& \quad \times \int_{-L_{p}}^{L_{n}} k_{n p}\left(L_{p}+x\right)\left\{\left[\alpha_{n 2}+n_{1}(x, t)\right]\left[\alpha_{p 2}+p_{1}(x, t)\right]-n_{0} p_{0}\right\} d x d t+\int_{0}^{\Theta}(\Theta-t) \int_{-L_{p}}^{L_{n}}\left(L_{p}+x\right) \times \\
& \times \alpha_{n 2} \frac{\partial \varphi_{h}(x, t)}{\partial x} d x d t+\int_{0}^{\Theta}(\Theta-t) \int_{-L_{p}}^{L_{n}} D_{n}\left[\alpha_{n 2}+n_{1}(x, t)\right] d x d t-\left(L_{n}+L_{p}\right) \int_{0}^{\Theta}(\Theta-t) n_{n}(t) \times \\
& \times D_{n}\left(L_{n}\right) d t+\alpha_{n 2} \hat{B}_{n 10}+\int_{0}^{\Theta} \int_{0}^{L_{n}}\left(L_{n}-x\right)\left[\alpha_{n 2}+n_{1}(x, t)-n_{0}(x)\right] d x d t-\left(L_{n}+L_{p}\right) \hat{G}_{11}+\frac{1}{2} I_{n 2}-
\end{aligned}
$$




$$
\begin{aligned}
& \left.-\frac{1}{2} J_{n 2}+\int_{0}^{\Theta}(\Theta-t) \int_{-L_{p}}^{L_{n}}\left[\alpha_{n 2}+n_{1}(x, t)\right] \frac{\partial D_{n}}{\partial x} d x d t+\frac{\left(L_{n}-L_{p}\right)^{2}}{2\left(L_{p}+L_{n}\right)}\right) \frac{e}{\varepsilon \varepsilon_{0}} \int_{0}^{\Theta}(\Theta-t) \int_{-L_{p}}^{L_{n}}\left(L_{n}-x\right) \times \\
& \times \mu_{n}\left[\alpha_{n 2}+n_{1}(x, t)\right]\left[\alpha_{p 2}+p_{1}(x, t)-\alpha_{n 2}-n_{1}(x, t)\right] d x d t+\hat{G}_{10}+\frac{1}{L_{p}+L_{n}} \int_{0}^{\Theta}\left\{\varphi_{k}+U(t)-\right. \\
& -\frac{e}{\varepsilon \varepsilon_{0}} \int_{-L_{p}}^{L_{n}}\left(L_{n}-x\right)\left[\alpha_{p 2}+p_{1}(x, t)-\alpha_{n 2}-n_{1}(x, t)\right] d x \int_{0}^{t} \int_{0-L_{p}}^{L_{n}} \mu_{n}\left[\alpha_{n 2}+n_{1}(x, \tau)\right] d x d \tau d t- \\
& -\int_{0}^{\Theta}(\Theta-t) \int_{-L_{p}}^{L_{n}} k_{n p}\left(L_{p}+x\right)\left\{\left[\alpha_{n 2}+n_{1}(x, t)\right]\left[\alpha_{p 2}+p_{1}(x, t)\right]-n_{0} p_{0}\right\} d x d t-\int_{0}^{\Theta}(\Theta-t) n_{n}(t) \times \\
& \times D_{n}\left(L_{n}\right) d t+\int_{0}^{\Theta}(\Theta-t) D_{n}\left(-L_{p}\right) n_{p}(t) d t+\int_{0}^{\Theta} \int_{0}^{L_{n}}\left(L_{n}-x\right)\left[\alpha_{n 2}+n_{1}(x, t)-n_{0}(x)\right] d x d t- \\
& -I_{n 1}+\alpha_{n 2} \hat{B}_{n 10}+\hat{B}_{n 11}+\int_{0}^{\Theta}(\Theta-t) \int_{-L_{p}}^{L_{n}}\left[\alpha_{n 2}+n_{1}(x, t)\right] \frac{\partial D_{n}}{\partial v} d x d t-\int_{0}^{\Theta} \int_{-L_{p}}^{0}\left(L_{p}+x\right)\left[n_{1}(x, t)+\right. \\
& \left.\left.+\alpha_{n 2}-n_{0}(x)\right] d x d t\right\}=0 \text {, } \\
& \frac{1}{2} \hat{G}_{12}+\frac{e}{\varepsilon \varepsilon_{0}} \int_{0}^{\Theta}(\Theta-t) \int_{-L_{p}}^{L_{n}}\left[\alpha_{p 2}+p_{1}(x, t)-\alpha_{n 2}-n_{1}(x, t)\right]\left[\alpha_{n 2}+n_{1}(x, t)\right]\left(L_{p}+x\right)\left(L_{n}-x\right) \times \\
& \times \mu_{n} d x d t-\frac{1}{2} \int_{0}^{\Theta} \int_{0}^{L_{n}}\left(L_{n}-x\right)\left[\alpha_{n 2}+n_{1}(x, t)\right] d x-L_{p}^{2} \frac{\Theta}{4}-\frac{1}{2} J_{n 1}-\frac{1}{L_{p}+L_{n}} \int_{0}^{\Theta}\left\{U(t)-\frac{e}{\varepsilon \varepsilon_{0}} \times\right. \\
& \times \int_{-L_{p}}^{L_{n}}\left(L_{n}-v\right)\left[\alpha_{p 2}+p_{1}(v, t)-\alpha_{n 2}-n_{1}(v, t)\right] d v+\varphi_{k} \int_{-L_{p}}^{L_{n}}\left(L_{p}+x\right)\left[\alpha_{n 2}+n_{1}(x, \tau)\right] \times \\
& \times \mu_{n} d x d \tau d t+\frac{1}{2} \int_{0}^{\Theta}(\Theta-t) \int_{-L_{p}}^{L_{n}} k_{n p}\left(L_{p}+x\right)\left\{\left[\alpha_{n 2}+n_{1}(x, t)\right]\left[\alpha_{p 2}+p_{1}(x, t)\right]-n_{0} p_{0}\right\} d x d t+ \\
& -\left(L_{n}+L_{p}\right) \int_{0}^{\Theta}(\Theta-t) D_{n}\left(L_{n}\right) n_{n}(t) d t+\int_{0}^{\Theta} \int_{0}^{L_{n}}\left(L_{n}-x\right)\left[\alpha_{n 2}+n_{1}(x, t)-n_{0}(x)\right] d x d t+\frac{1}{2} I_{n 2}+ \\
& +\int_{0}^{\Theta}(\Theta-t) \int_{-L_{p}}^{L_{n}}\left[\alpha_{n 2}+n_{1}(x, t)\right] \frac{\partial D_{n}}{\partial x} d x d t+\int_{0}^{\Theta}(\Theta-t) \int_{-L_{p}}^{L_{n}} D_{n}\left[\alpha_{n 2}+n_{1}(x, t)\right] d x d t+\alpha_{n 2} \hat{B}_{n 10}+ \\
& +\frac{\left(L_{n}-L_{p}\right)^{2}}{2\left(L_{p}+L_{n}\right)} \int_{0}^{\Theta}(\Theta-t) \int_{-L_{p}}^{L_{n}}\left(L_{n}-x\right) \mu_{n}\left[\alpha_{p 2}+p_{1}(x, t)-\alpha_{n 2}-n_{1}(x, t)\right]\left[\alpha_{n 2}+n_{1}(x, t)\right] d x d t \times
\end{aligned}
$$




$$
\begin{aligned}
& \times \frac{e}{\varepsilon \varepsilon_{0}}+\hat{B}_{n 11}-\frac{1}{2} J_{n 2}+\hat{G}_{21}+\int_{0}^{\Theta}\left\{U(t)-\frac{e}{\varepsilon \varepsilon_{0}} \int_{-L_{p}}^{L_{n}}\left(L_{n}-x\right)\left[\alpha_{p 2}+p_{1}(x, t)-\alpha_{n 2}-n_{1}(x, t)\right] d x+\right. \\
& \left.+\varphi_{k}\right\} \frac{1}{L_{p}+L_{n}} \int_{0}^{t} \int_{-L_{p}}^{L_{n}} \mu_{n}\left[\alpha_{n 2}+n_{1}(x, \tau)\right] d x d \tau d t-\left(L_{n}+L_{p}\right) \hat{G}_{11}-\int_{0}^{\Theta}(\Theta-t) D_{n}\left(L_{n}\right) n_{n}(t) d t+ \\
& +\int_{0}^{\Theta}(\Theta-t) n_{p}(t) D_{n}\left(-L_{p}\right) d t-\int_{0-L_{p}}^{\Theta} k_{n p}^{L_{n}}\left(L_{p}+x\right)\left\{\left[\alpha_{n 2}+n_{1}(x, t)\right]\left[\alpha_{p 2}+p_{1}(x, t)\right]-n_{0} p_{0}\right\} d x \times \\
& \times(\Theta-t) d t-\int_{0-L_{p}}^{\Theta}\left(L_{p}+x\right)\left[\alpha_{n 2}+n_{1}(x, t)\right] d x d t+\int_{0}^{\Theta}(\Theta-t) \int_{-L_{p}}^{L_{n}} \frac{\partial D_{n}}{\partial v}\left[\alpha_{n 2}+n_{1}(x, t)\right] d x d t- \\
& \left.-J_{n 1}+\alpha_{n 2} \hat{B}_{n 10}+\hat{B}_{n 11}-\hat{G}_{11}\right\}=0 .
\end{aligned}
$$

Parameters $b_{i}$ are determined by the following relations

$$
\begin{aligned}
& b_{1}=M_{n 00 n p 11}+M_{n 00 n p 11}+\frac{1}{2}\left(L_{n}-L_{p}\right)^{2} M_{n 00 n p 10}+M_{n 00 n p 00}\left(L_{n}-L_{p}\right)^{4} / 4\left(L_{p}+L_{n}\right)^{2} \\
& \text { where } M_{\rho i j \rho \rho k l}=\frac{e}{\varepsilon \varepsilon_{0}} \int_{0}^{\Theta}(\Theta-t) \int_{-L_{p}}^{L_{n}} \mu_{\rho}\left(L_{p}+x\right)^{k}\left(L_{n}-x\right)^{l} \rho_{1}^{i}(x, t) \rho_{1}^{j}(x, t) d x d t \\
& b_{2}=M_{n 00 n p 01} \frac{\left(L_{n}-L_{p}\right)^{2}}{2\left(L_{p}+L_{n}\right)}-M_{n 00 n p 00} \frac{\left(L_{n}-L_{p}\right)^{4}}{4\left(L_{p}+L_{n}\right)^{2}}-\frac{2 L_{p} L_{n} N_{1000}}{\left(L_{p}+L_{n}\right)^{2}}-M_{n 00 n p 10} \frac{\left(L_{n}-L_{p}\right)^{2}}{2\left(L_{n}+L_{p}\right)}+ \\
& +M_{n 00 n p 11} \text {, } \\
& \text { where } N_{i j k l}=\int_{0}^{\Theta}(\Theta-t) \int_{-L_{p}}^{L_{n}} k_{n p}\left(L_{p}+x\right)^{i}\left(L_{n}-x\right)^{j} n_{1}^{k}(x, t) p_{1}^{l}(x, t) d x d t \\
& b_{3}=M_{n 01 n p 11}-M_{n 10 n p 11}-M_{n 10 n p 11}+\frac{e P_{n 1 p 11 n 0}}{\varepsilon \varepsilon_{0}\left(L_{p}+L_{n}\right)}-\frac{1}{L_{p}+L_{n}} \int_{0}^{\Theta}\left[\varphi_{k}+U(t)\right] \int_{0}^{t} \int_{L_{n}}^{x} \mu_{n} d v d \tau d t+ \\
& +\frac{N_{1001}}{2}+\int_{0}^{\Theta}(\Theta-t)\left[D_{n}\left(L_{n}\right)-D_{n}\left(-L_{p}\right)\right] d t-M_{n 10 n p 10} \frac{\left(L_{n}-L_{p}\right)^{2}}{2\left(L_{n}+L_{p}\right)}+\frac{\left(L_{n}-L_{p}\right)^{2}}{2\left(L_{p}+L_{n}\right)^{2}} \int_{0}^{\Theta}\left[\varphi_{k}+U(t)\right] \times \\
& \times \int_{0}^{t} \int_{-L_{p}}^{L_{n}} \mu_{n} d x d \tau d t+\left(M_{n 01 n p 01}-M_{n 10 n p 01}\right) \frac{\left(L_{n}-L_{p}\right)^{2}}{2\left(L_{p}+L_{n}\right)}-M_{n 10 n p 01} \frac{\left(L_{n}-L_{p}\right)^{2}}{2\left(L_{p}+L_{n}\right)}+M_{n 10 n p 00} \times \\
& \times \frac{\left(L_{n}-L_{p}\right)^{4}}{4\left(L_{p}+L_{n}\right)^{2}}+\hat{B}_{n 10}+\frac{\left(L_{n}-L_{p}\right)^{2}}{2\left(L_{p}+L_{n}\right)^{2}} \int_{0}^{\Theta}(\Theta-t)\left[D_{n}\left(L_{n}\right)-D_{n}\left(-L_{p}\right)\right] d t-\left(P_{n 1 p 10}-P_{n 1 n 10}\right) \times \\
& \times \frac{e}{\varepsilon \varepsilon_{0}} \frac{\left(L_{n}-L_{p}\right)^{2}}{2\left(L_{p}+L_{n}\right)^{2}}-N_{1001} \frac{\left(L_{n}-L_{p}\right)^{2}}{2\left(L_{p}+L_{n}\right)^{2}}+\Theta \frac{L_{n}^{2}}{4}-\Theta L_{p}^{2} \frac{3\left(L_{n}-L_{p}\right)^{2}}{2\left(L_{p}+L_{n}\right)^{2}}+\hat{B}_{n 10} \frac{\left(L_{n}-L_{p}\right)^{2}}{2\left(L_{p}+L_{n}\right)^{2}}+
\end{aligned}
$$




$$
+\Theta L_{n}^{2}\left(L_{n}-L_{p}\right)^{2} / 4\left(L_{p}+L_{n}\right)^{2}
$$

where;

$$
\begin{aligned}
& b_{4}=M_{n 10 n p 01} \frac{\left(L_{n}-L_{p}\right)^{2}}{2\left(L_{p}+L_{n}\right)}-M_{n 10 n p 10} \frac{\left(L_{n}-L_{p}\right)^{2}}{2\left(L_{n}+L_{p}\right)}-M_{n 10 n p 00} \frac{\left(L_{n}-L_{p}\right)^{4}}{2\left(L_{p}+L_{n}\right)^{2}}+\frac{2 L_{n} L_{p} N_{1010}}{\left(L_{p}+L_{n}\right)^{2}}+ \\
& +M_{n 10 n p 10} \text {; } \\
& b_{5}=M_{n 11 n p 11}-M_{n 20 n p 11}-\frac{1}{2} \int_{0}^{\Theta} \int_{0}^{L_{n}}\left(L_{n}-x\right) n_{1}(x, t) d x-\frac{1}{2} \int_{0}^{\Theta} \int_{-L_{p}}^{0}\left(L_{p}+x\right) n_{1}(x, t) d x-\frac{1}{L_{p}+L_{n}} \times \\
& \times \int_{0}^{\Theta}\left[\varphi_{k}+U(t)\right] \int_{0}^{t} \int_{-L_{p}}^{L_{n}} \mu_{n}\left(L_{p}+x\right) n_{1}(x, \tau) d x d \tau d t+\frac{1}{2} \hat{G}_{12}-\frac{1}{L_{p}+L_{n}} \frac{e P_{n 1 n 11}}{\varepsilon \varepsilon_{0}}+\frac{e P_{n 11 p 11 n 1}}{\left(L_{p}+L_{n}\right) \varepsilon \varepsilon_{0}}- \\
& -\frac{n_{0} p_{0} N_{1000}}{2}-\left(L_{n}+L_{p}\right) \int_{0}^{\Theta}(\Theta-t) D_{n}\left(L_{n}\right) n_{n}(\tau) d \tau+\frac{N_{1011}}{2}+\hat{B}_{n 11}+\frac{1}{2} I_{n 2}-\frac{1}{2} J_{n 2}-\frac{1}{2} I_{n 1}+ \\
& +\int_{0}^{\Theta} \int_{0}^{L_{n}}\left(L_{n}-x\right) n_{1}(x, t) d x d t+\int_{0}^{\Theta}(\Theta-t) \int_{-L_{p}}^{L_{n}} D_{n} n_{1}(x, t) d x d t+\int_{0}^{\Theta}(\Theta-t) \int_{-L_{p}}^{L_{n}} n_{1}(x, t) \frac{\partial D_{n}}{\partial x} d x d t+ \\
& +G_{21} \frac{\left(L_{n}-L_{p}\right)^{2}}{2\left(L_{p}+L_{n}\right)}-\left(L_{n}+L_{p}\right) G_{11}+M_{n 11 n p 01}-\frac{\left(L_{n}-L_{p}\right)^{2}}{2\left(L_{p}+L_{n}\right)} \int_{0}^{\Theta}(\Theta-t) D_{n}\left(L_{n}\right) n_{n}(t) d t ; \\
& b_{6}=\frac{2 e P_{p 1 n 01 p 0}+2 \varepsilon \varepsilon_{0}\left(L_{p}+L_{n}\right) M_{p 00 n p 11}-\varepsilon \varepsilon_{0} M_{p 00 n p 00} L_{n}^{2}+\varepsilon \varepsilon_{0}\left(L_{n}-L_{p}\right)^{2} M_{p 00 p 01}}{2 \varepsilon \varepsilon_{0}\left(L_{p}+L_{n}\right)} ; \\
& b_{7}=\left(N_{1000}-M_{p 00 n p 01}\right) \frac{\left(L_{n}-L_{p}\right)^{2}}{2\left(L_{n}+L_{p}\right)}-\frac{e P_{p 1 n 0 n 1 p 1}}{\varepsilon \varepsilon_{0}\left(L_{p}+L_{n}\right)}-L_{n}^{2} \frac{M_{p 00 n p 00}}{2\left(L_{p}+L_{n}\right)}-N_{1100}-M_{p 00 n p 11} \text {; } \\
& b_{8}=M_{p 01 n p 11}-M_{p 10 n p 11}-\frac{1}{L_{p}+L_{n}} \int_{0}^{\Theta}(\Theta-t)\left[\varphi_{k}+U(t)\right] \int_{0}^{t} \int_{-L_{p}}^{L_{n}}\left(L_{p}+x\right) \mu_{p} d x d \tau d t+ \\
& +\frac{1}{L_{p}+L_{n}} \frac{e}{\varepsilon \varepsilon_{0}} \int_{0}^{\Theta}(\Theta-t) \int_{0}^{t} \int_{-L_{p}}^{L_{n}}\left(L_{n}-x\right)\left[p_{1}(x, \tau)-n_{1}(x, \tau)\right] d x \int_{-L_{p}}^{L_{n}}\left(L_{p}+x\right) \mu_{p} d x d \tau d t- \\
& -\frac{5}{6} \Theta L_{p}^{3}+\frac{e L_{n}^{2} M_{p 01 n p 10}}{4 \varepsilon \varepsilon_{0}\left(L_{p}+L_{n}\right)}-\hat{B}_{p 10}+L_{n}^{2} L_{p} \frac{\Theta}{2}+2 \int_{0}^{\Theta}(\Theta-t)\left[D_{p}\left(L_{n}\right)-D_{p}\left(-L_{p}\right)\right] d t+ \\
& +M_{p 01 n p 11}-\frac{e}{\varepsilon \varepsilon_{0}} \frac{1}{L_{p}+L_{n}} \int_{0}^{\Theta} \int_{-L_{p}}^{L_{n}}\left(L_{n}-x\right)\left[p_{1}(x, t)-n_{1}(x, t)\right] d x \int_{0}^{t} \int_{-L_{p}}^{L_{n}} \mu_{p} d v d \tau d t+\Theta \frac{L_{n}^{3}}{3}+ \\
& \times+2 \int_{0}^{\Theta}(\Theta-t)\left[D_{p}\left(L_{n}\right)-D_{p}\left(-L_{p}\right)\right] d t+\frac{1}{L_{p}+L_{n}} \int_{0}^{\Theta}\left[\varphi_{k}+U(t)\right] \int_{0}^{t} \int_{-L_{p}}^{L_{n}} \mu_{p} d x d \tau d t-N_{1110}+
\end{aligned}
$$




$$
\begin{aligned}
& +\frac{\left(L_{n}-L_{p}\right)^{2}}{2\left(L_{n}+L_{p}\right)}\left\{\int_{0}^{\Theta}(\Theta-t)\left[D_{p}\left(L_{n}\right)-D_{p}\left(-L_{p}\right)\right] d t+\frac{\Theta}{2}\left(L_{n}^{2}-L_{p}^{2}\right)\left(M_{p 01 n p 01}-M_{p 10 n p 01}\right)+\right. \\
& \left.+2 M_{p 01 n p 01}-2 M_{p 10 n p 01}+\hat{B}_{p 10}+\hat{B}_{p 10}+N_{1010}\right\} \text {; } \\
& b_{9}=\frac{2\left(L_{n}-L_{p}\right)^{2} N_{1001}-4\left(N_{1101}+M_{p 01 n p 11}\right)\left(L_{n}+L_{p}\right)-L_{n}^{2} M_{p 01 n p 10}-2\left(L_{n}-L_{p}\right)^{2} M_{p 01 n p 01}}{4\left(L_{n}+L_{p}\right)} \\
& b_{10}=\frac{1}{2} \hat{G}_{12}+\frac{1}{2} \hat{G}_{2}-\frac{1}{L_{p}+L_{n}} \int_{0}^{\Theta}(\Theta-t)\left[\varphi_{k}+U(t)\right] \int_{0}^{t} \int_{-L_{p}}^{L_{n}}\left(L_{p}+x\right) \mu_{p} p_{1}(x, \tau) d x d \tau d t- \\
& -\frac{1}{2} \int_{0}^{\Theta} \int_{0}^{L_{n}}\left(L_{n}-x\right)^{2} p_{1}(x, t) d x d t+\frac{e}{\varepsilon} \frac{\left(P_{p 1 p 11 p 1}-P_{p 1 p 11 n 1}\right)}{\varepsilon_{0}\left(L_{p}+L_{n}\right)}+M_{p 02 n p 11}-N_{1111}-\hat{B}_{p 11}+ \\
& +\frac{1}{2} \int_{0}^{\Theta} \int_{-L_{p}}^{0}\left(L_{p}-x\right)^{2} p_{1}(x, t) d x d t-M_{p 11 n p 11}+\left(L_{p}+L_{n}\right) \int_{0}^{\Theta} \int_{0}^{L_{n}}\left(L_{n}-x\right) p_{1}(x, t) d x d t+N_{1100} \times \\
& \times p_{0} n_{0}+\int_{0}^{\Theta}(\Theta-t) \int_{-L_{p}}^{L_{n}} p_{1}(x, t) \frac{\partial D_{p}}{\partial x} d x d t+\frac{1}{L_{p}+L_{n}} \int_{0}^{\Theta}\left[\varphi_{k}+U(t)\right] \int_{0}^{t} \int_{-L_{p}}^{L_{n}} \mu_{p} p_{1}(v, \tau) d v d \tau d t- \\
& -\int_{0}^{\Theta}(\Theta-t) D_{p}\left(L_{n}\right) p_{n}(t) d t+\hat{B}_{p 11}-\frac{\left(L_{n}-L_{p}\right)^{2}}{2\left(L_{n}+L_{p}\right)} \int_{0}^{\Theta}(\Theta-t) D_{p}\left(L_{n}\right) p_{n}(t) d t+\frac{\left(L_{n}-L_{p}\right)^{2}}{2\left(L_{n}+L_{p}\right)} \times \\
& \times \int_{0}^{\Theta}(\Theta-t) \int_{-L_{p}}^{0}\left(L_{p}+x\right) G d x d t+\frac{\left(L_{n}-L_{p}\right)^{2}}{2\left(L_{n}+L_{p}\right)} \int_{0}^{\Theta}(\Theta-t) \int_{-L_{p}}^{L_{n}} p_{1}(x, t) \frac{\partial D_{p}}{\partial x} d x d t-\frac{\left(L_{n}-L_{p}\right)^{2}}{2\left(L_{n}+L_{p}\right)} \times \\
& \times \int_{0}^{\Theta}(\Theta-t) \int_{0}^{L_{n}}\left(L_{n}-x\right) G d x d t-G_{11}+\frac{\left(L_{n}-L_{p}\right)^{2}}{2\left(L_{n}+L_{p}\right)}\left[\int_{0}^{\Theta}(\Theta-t) D_{p}\left(-L_{p}\right) p_{p}(t) d t-\int_{0}^{\Theta} \int_{-L_{p}}^{0} p_{1}(x, t) \times\right. \\
& \left.\times\left(L_{p}+x\right) d x d t+\left(N_{1011}-n_{0} p_{0} N_{1000}\right)\right] .
\end{aligned}
$$

\section{Acknowledgements}

This work is supported by the contract 11.G34.31.0066 of the Russian Federation Government and educational fellowship for scientific research.

\section{REFERENCES}

[1] V.I. Lachin, N.S. Savelov. Electronics. Rostov-on-Don: Phoenix. 2001. 446 p.
[2] A.B. Grebene. Bipolar and MOS analogous integrated circuit design. New York, John Wyley and Sons, 1983, 894 p.

[3] Z.Yu. Gotra. Technology of electronic devices. Moscow: Radio and Communication. 1991. 528 p.

[4] K.V. Shalimova. Physics of semiconductors. Moscow: Energoatomizdat. 1985. 392 p.

[5] A.G. Alexenko, I.I. Shagurin. Microcircuitry. Moscow: Radio and Communication. 1990. 496 p.

[6] E.L. Pankratov. Phys. Rev. B. Vol.72 (7). P. 075201 (2005).

[7] E.L. Pankratov. Russian Microelectronics. Vol. 36 (1). P. 33 (2007).

[8] E.L. Pankratov. J. Comp. Theor. Nanoscience. Vol. 8 (2). P. 
207 (2011).

[9] E.L. Pankratov, E.A. Bulaeva. Reviews in Theoretical Science. Vol. 1 (1). P. 58 (2013).

[10] E.L. Pankratov, E.A. Bulaeva. J. Comp. Theor. Nanoscience. Vol. 10 (4). P. 888 (2013).

[11] Yu.D. Sokolov. Applied Mechanics. Vol. 1. P. 23 (1955).

[12] E.L. Pankratov. J. Appl. Phys. V.101 (11). P. 114308 (2007).

[13] E.V. Kalinina, V.G. Kossov, R.R.Yafaev, A.M. Strel'chuk, G.N. Violina. Semiconductors. V.44 (6). P. 807-816 (2010) 